\title{
VALIDACION DE UNA ESCALA DE CALIDAD DE VIDA EN UN GRUPO DE PERSONAS CON ESQUIZOFRENIA DE LA REGION METROPOLITANA - CHILE
}

\author{
VALIDATION OF A SCALE OF QUALITY OF LIFE IN PEOPLE WITH \\ SCHIZOPHRENIA IN THE METROPOLITAN REGION - CHILE
}

\author{
ANGELINA D OIS CASTELLON*, AIXA CONTRERAS MEJIAS**,

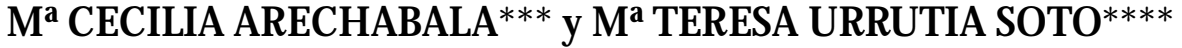

\begin{abstract}
RESUMEN
El estudio evalúa las propiedades psicométricas de la versión española de la Short form-36 H ealth Survey (SF36), diseñada por el Medical Outcomes Study y la validada por Alonso (1999) en personas con esquizofrenia crónica. Para esto, se realizó una aplicación piloto que mostró la necesi dad de realizar adaptaciones en el instrumento. Luego, el instrumento adaptado fueaplicado en la Región M etropolitana a 99 sujetos con esquizofrenia. Se utilizó el programa SPSS para el análisis estadístico, el cual mostró que el comportamiento de los ítemes permite aplicarla en una versión modificada.
\end{abstract}

Palabras claves: Calidad de vida, esquizofrenia, escala de evaluación.

\begin{abstract}
The study evaluates the psychometric properties of the spanish version of the Short form- $36 \mathrm{H}$ ealth Survey (SF36), in people with schizophrenia. The SF-36 inventory designed by the M edical Outcomes Study and the one validated by Alonso (1999) wereused. With this purpose, a pilot application was carried out, showing that it was necessary to make some adaptations to the instrument. Then, the adapted instrument was applied in ninety nine person with schizophrenia from the Metropolitan Region. The program SPSS used for the statistical analysis showed that the behaviour of items allowed to apply it in a modified version.
\end{abstract}

Keywords: Quality of life, schizophrenia, inventory of mental health.

Fecha recepción: 20/11/06. Fecha aceptación: 02/05/07.

\section{INTRODUCCION}

La Organización M undial de la Salud (1980) define calidad de vida como "la percepción que tienen los individuos acerca de su posi- ción en la vida, teniendo en cuenta el contexto del sistema cultural y de valores en el que viven y en relación con sus metas, expectativas, normas e intereses". Esta aproximación enfatiza sobre los aspectos personales, resal-

\footnotetext{
* Enfermera-matrona, Terapeuta Familiar y de Parejas, Profesora Auxiliar Departamento de Salud del Adulto y Adulto M ayor, Escuela de Enfermería, Pontificia Universidad Católica deChile. E-mail: adois@uc.cl

** Enfermera-matrona, Magíster en Psicología Social y Comunitaria, Profesora Adjunta Departamento de Salud de la Mujer, Escuela de Enfermería, Pontificia Universidad Católica deChile. E-mail: acontrem@uc.cl

*** Enfermera, Especialista en Enfermería Geronto-Geriátrica M agíster en Psicología Social y Comunitaria, Profesora Adjunta Departamento de Salud del Adulto y Adulto M ayor, Escuela deEnfermería, PontificiaUniversidad CatólicadeChile. E-mail: marechab@uc.cl

**** Enfermera-matrona, Magíster en Nutrición, Profesora Adjunta Departamento de Salud de la M ujer, Escuela de Enfermería, Pontificia Universidad Católica deChile. E-mail: murrutis@uc.cl
} 
tando así la individualidad y unicidad del constructo.

Posteriormente, Borthwick-Duffy (1992) y Felce y Perry (1995) incorporan al concepto de calidad de vida, una serie de dominios según los cuales ésta sería definida como "la calidad de las condiciones devida deuna persona, la satisfacción experimentada por esa persona con dichas condiciones vitales, la combinación deestos componentes objetivos y subjetivos y por último, como la combinación de las condiciones de vida y satisfacción personal ponderadas por la escala de valores, aspiraciones y expectativas personales". Esta perspectiva incorpora a la dimensión subjetiva del concepto de calidad de vida, elementos objetivos como es la calidad de las condiciones de vida de las personas.

M ajali Mahasneh (2001) señala que la calidad devida de una persona serelaciona más con la percepción que ésta tiene de su estado, quecon el curso propiamentetal desu enfermedad y adicionalmentecuando laspersonas se perciben a sí mismas con una mejor calidad devida se refuerzan sus capacidades para hacerse cargo de su salud, mejora su autoestima y los motiva a realizar actividades en pro de una vida más satisfactoria, lo que concuerda con lo encontrado por Björkman y Hansson (2002). Sin embargo, otros autores (Padierna, Quintana, Arostegui, González, H orcajo y Etxebarría, 2000) señalan que la percepción decalidad de vida se relaciona con el curso de la enfermedad y las características de su tratamiento, lo que es confirmado por el estudio de Delamillieure, Ochoa-Torres, Vasse, Brazo, Gourevitch, Langlois et al. (2005) que hace especial mención a la ausencia de sintomatología negativa en la percepción de la calidad de vida.

Por otro lado, Dernovzek, Prevolnik Rupel, Rebolj y Tavcar (2002) afirman quelos principales factores que intervienen en la calidad de vida de las personas con esquizofrenia son el rendimiento psicosocial y los efectossecundarios de los tratamientos: estos hallazgos concuerdan con lo encontrado por Caron,
Lecomte, Stip y Renaud (2005), quienes plantean que los mejores predictores de la calidad de vida en esta población son el soporte social, los mecanismos de afrontamiento el nivel de educación y el número de hospitalizaciones previas.

Giner, Ibáñez, Cervera, San M artín y Caballero (2001), en su estudio sobre calidad de vida de las personas con esquizofrenia, concluyen que en ésta intervienen de forma negativa las dificultades en las relaciones interpersonales y las dificultades cognitivasy afectivas, que pueden interpretarse como partede la experiencia subjetiva del enfermo. En el mismo estudio se demostró que el enfermo esquizofrénico es capaz de diferenciar entre aquellos aspectos de su vida que considera como positivos o favorables, de aquellos que le producen un auténtico sufrimiento personal y que afectan su calidad de vida. Dentro de estas experiencias se puede mencionar la percepción de discriminación asociada al estigma de la enfermedad (Katschnig, 2000) y la institucionalización prolongada (Cavieres y Valdevenito, 2005).

Considerando estosaspectosy otras variables que intervienen en la calidad de vida de las personas con esquizofrenia, el M inisterio de Salud a través del Plan Nacional de Salud Mental (Ministerio de Salud [MINSAL], 2000) ha establecido dentro de sus objetivos principales la rehabilitación del pacientemental y su pronta reinserción social ya que, en general, todas las variables subjetivas deautopercepción desalud y bienestar general muestran asociación significativa a un riesgo mayor de discapacidad cuando refieren calificaciones negativas o mayor insatisfacción (Acuña y Rojas, 2000).

La investigación sobre percepción de calidad de vida en personas con problemas de salud mental se torna, entonces, altamenterelevante, ya que puede constituirse como una categoría organizadora y de referencia al momento de establecer cuidados de enfermería y objetivos terapéuticos. Este hecho demuestra que se hace necesario contar con instrumen- 
tosqueevalúen la calidad devida, especialmente val idados para este tipo de poblaciones.

La presente investigación pretende analizar el proceso de validación del SF-36 como instrumento demedición dela calidad devida de personas con esquizofrenia y sugerir las modificaciones necesarias para su utilización en esta población en particular.

\section{Instrumento}

Short form-36 Health Survey (SF-36) fue diseñado por el M edical Outcomes Study para medir percepción decalidad devida, y ha sido validado en diferentes grupos poblacionales cumpliendo con criterios de relevancia y validez (Alonso, 1999; Jenkinson, Wright, Coulter, 1994; M cH orney, Ware, Raczek, 1993; Brazier y Harper, 1992).

El test detecta tanto estados positivos como negativos desalud. El contenido delosítemes se centra en el estado funcional y el bienestar emocional. Su ámbito de aplicación abarca estudios descriptivos y de evaluación.

Consta de 36 ítemes, que exploran 8 dimensiones del estado de salud: función física, función social, limitaciones del rol: problemas físicos, problemasemocionales, salud mental, vitalidad, dolor y percepción de la salud general. Existe una cuestión, no incluida en estas ocho categorías, que explora los cambios experimentados en el estado de salud en el último año.

Warey Sherbourneen 1992 reportaron un índice de confiabilidad (Alpha Cronbach's) $>0.85$, coeficiente de relevancia $>0.75$ para todas las dimensiones, excepto para el funcionamiento social y por validez de constructo en términosdela distinción entre gruposcon diferentes expectativas de vida.

La versión en español fue adaptada por Alonso en 1999 y la validez de la versión española ha sido analizada por Ayuso-M ateos, Lasa, Vázquez-Barquero, O viedo y DiezManeique(1999).

Se trata de un cuestionario autoadminis- trado, aunque también se ha utilizado mediante un entrevistador, a través del teléfono o mediante soporte informático.

El mismo instrumento fue validado para poblaciones con desordenes mentales severos por distintos investigadores (Reine, Simeoni, Auquier, Loundou , Aghababian y Lancon, 2005; Kebede, Alem, Shibre, Negash, Deyassa y Beyero, 2004; Tunis, Croghan, Heilman, Johnstone y O benchain, 1999).

\section{MATERIAL Y METODO}

\section{Participantes}

Para la validación del instrumento, se seleccionó intencionalmente una muestra formada por 99 personas con esquizofrenia crónica compensada, entre 35 y 75 años de edad, que no presentaran una patología orgánica crónica descompensada o invalidante concomitante a la esquizofrenia, pertenecientes a las comunas de Independencia y Recoleta, en control y tratamiento en el Instituto Psiquiátrico José Horwitz Barak de Santiago, que viven en HogaresProtegidos o Unidades deLargaEstadía. El 88\% dela muestra son mujeresy el $12 \%$ hombres con un promedio de edad de 54.3 años y una desviación estándar de 12.04

La primera etapa del proceso de validación del instrumento consistió en realizar la validación del contenido modificando el lenguaje de la versión española del SF-36 a un lenguaje del tipo coloquial ajustado a la realidad chilena de los enfermos mentales. Luego se sometió a validación de expertos del área de la salud mental y la psiquiatría, que evaluaron la representatividad y adecuación muestral del contenido del instrumento, en función del referente teórico y losindicadores de las variables que éste pretende medir.

La segunda etapa del proceso consistió en la aplicación de una prueba piloto del instrumento en una población con características similares a las que conformarían la muestra de estudio y que cumplieron con los criterios 
de inclusión. Este proceso se repitió cuatro veces a diferentes muestras que conservaron las características, asegurando que su contenido y la escala de respuesta, eran comprensibles y adecuados para la misma.

En este proceso se eliminaron dos ítemes que los usuarios no comprendían porque no seajustaban al tipo de vida queellos desarroIlan. Estas preguntas corresponden a la $\mathrm{N} 020$ “Durante las 4 últimas semanas, ihasta qué punto su salud física o los problemas emocionales han dificultado sus actividades sociales habituales con la familia, los amigos, los vecinos u otras personas?", y el ítem $\mathrm{N}$ 22: "Durante las 4 últimas semanas, ihasta qué punto el dolor le ha dificultado su trabajo habitual (incluido el trabajo fuera de casa y las tareas domésticas)?".

La escala de respuesta final también fue modificada a partir de una escala tipo Likert de 5 alternativas a una de tres, siguiendo las recomendaciones formuladas por los jueces, debido a la dificultad dela muestra para comprender la amplitud y diversidad de las respuestas propuestas en el instrumento original ya que autores como Hernández, Fernández y Baptista (1998) demuestran quela confiabilidad deun instrumento esindependiente del número de respuestas que tienen los ítemes.

Para evaluar la consistencia interna $(\mathrm{Cl}) 0$ coeficiente de homogeneidad se utilizó el coeficiente Alpha, que evalúa la correlación entre cada uno de los elementos y la totalidad del instrumento. El criterio de inclusión viene dado para aquellos elementos que presenten una correlación, mayor de 0.70 , con la totalidad del test, lo que indica que ese elemento mide lo mismo que el test en su conjunto.

Según lo planteado por Kerlinger (1975), dentro de los criterios psicométricos, el coeficiente al pha permite evaluar la cantidad de varianza sistemática - proporción de varianza común entre dos variables- en términos de su estabilidad, certeza, predictibilidad y exactitud.

Basados en los parámetros que seconsideran para el análisis (Cerda, 1984):
- Correlaciones entre 1.00 y 0.9 son significados como "muy alta".

- Correlaciones entre 0.9 y 0.7 son significados como "alta".

- Correlaciones entre 0.7 y 0.4 son significados como "moderada".

- Correlaciones entre 0.4 y 0.2 son significados como "baja".

- Correlaciones inferiores a 0.2 son significados como "muy baja".

Para fines de esta investigación se consideró aceptable un coeficiente de correlación mayor o igual a 0.70 .

\section{Aplicación del instrumento}

Pese a que el instrumento original fue diseñado para ser autoadministrado, por la naturaleza de la muestra en estudio éste fue aplicado por encuestadores capacitados para esos fines.

Previo a la aplicación del instrumento, a cada uno de los participantes, o en su defecto a su representantelegal, seles solicitó quefirmaran un Consentimiento Informado (anexo 1). En este formulario se daba a conocer el objetivo de la investigación, el tipo de colaboración que se esperaba de ellos, las condiciones de anonimato y el carácter voluntario de su participación. También se indicaba el correo electrónico y teléfono de contacto de las investigadoras responsables con el objeto que se pudiera dirigir cualquier comentario o duda sobre la investigación.

\section{ANALISIS DE LOS RESULTADOS}

La estimación de la consistencia interna de la escala se hizo a través del coeficiente Alpha de Cronbach. El resultado obtenido fue 0.72 para la población en estudio, lo que es considerado como alto de acuerdo a los criterios propuestos por Hernández et al. (1998).

El coeficienteAlphadeCronbach informado para las subescalas que mideel instrumento se encuentra en la Tabla $\mathrm{N}$ ㅇ: 
Tabla 1. Coeficientes al fa de las dimensiones del SF-36.

\begin{tabular}{|lc|}
\hline Variables & Alpha \\
\hline Limitación en actividad física por problemas de salud & 0.66 \\
Limitación en actividades propias del rol por problemas de salud física & 0.71 \\
Salud mental general (stress, bienestar) & 0.36 \\
Limitación en actividades propias del rol por problemas emocionales & 0.99 \\
Energía - vitalidad & 0.35 \\
Percepción general de salud & 0.71 \\
Limitación en actividades sociales por problemas físicos o emocionales & $*$ \\
Dolor & $*$ \\
\hline
\end{tabular}

(*) Ambas subescalas contienen una sola pregunta, por lo que no es posible obtener el coeficienteAlpha.

En la Tabla 1 se puede observar que la subescala "Limitación en actividades propias del rol por problemas emocionales" obtiene un coeficiente de confiabilidad muy alto; las subescalas, "Limitación en actividades propias del rol por problemas de salud física” y "Percepción general desalud" obtienen coeficien- tes de confiabilidad altos; y las sub escalas de Salud M ental - Energía y Vitalidad, coeficientes muy bajos.

En la Tabla 2 se muestra el análisis de la consistencia interna que incluye el valor del coeficiente alpha si el ítem es eliminado.

Tabla 2. Consistencia interna por ítemes eliminados.

\begin{tabular}{|l|c|c|}
\hline Escala & $\begin{array}{c}\text { Item- total } \\
\text { correlation }\end{array}$ & $\begin{array}{c}\text { Alpha if } \\
\text { Item } \\
\text { Deleted }\end{array}$ \\
\hline En general usted diría que su salud es & .2709 & .7128 \\
Cómo diría que es su salud actual comparada con la de hace un año & .3202 & .7101 \\
Por su salud actual, ¿se le hace difícil hacer esfuerzos intensos tales como: correr, levantar & & \\
$\quad$ objetos pesados & .3324 & .7086 \\
Por su salud actual, ise le hace difícil hacer esfuerzos moderados como: mover una mesa, & & \\
$\quad$ como pasar la enceradora o caminar más de1 hora? & .3104 & .7107 \\
Por su salud actual, ise le hace difícil levantar o llevar la bolsa de la compra? & .2581 & .7130 \\
Por su salud actual, ise le hace difícil subir varios pisos por la escalera? & .1123 & .7216 \\
Por su salud actual, ¿se le hace difícil subir 1 solo piso por la escalera? & .6013 & .6930 \\
Por su salud actual, ¿se le hace difícil agacharse o arrodillarse? & .1660 & .7408 \\
Por su salud actual, ¿se le hace difícil caminar 1 cuadra? & .3712 & .7063 \\
Por su salud actual, ise le hace difícil caminar 2 cuadras? & .4744 & .7048 \\
Por su salud actual, ise le hace difícil caminar 4 o más cuadras? & .7316 & .6981 \\
Por su salud actual, ise le hace difícil bañarse o vestirse solo? & .4708 & .7057 \\
Durante el último mes tuvo que reducir el tiempo dedicado al trabajo o a sus actividades & & \\
$\quad$ diarias a causa de su salud física & .4801 & .7036 \\
Durante el último mes hizo menos actividades de lo que hubiera querido hacer a causa de & & \\
$\quad$ su salud física & .5647 & .7038 \\
\hline
\end{tabular}


Tabla 2 (Continuación).

\begin{tabular}{|l|c|c|}
\hline Escala & $\begin{array}{l}\text { Item- total } \\
\text { correlation }\end{array}$ & $\begin{array}{c}\text { Alpha if } \\
\text { Item } \\
\text { Deleted }\end{array}$ \\
\hline Durante el último mes tuvo que dejar de hacer al gunos de sus quehaceres diarios a causa & & \\
$\quad$ de su salud física & -.3187 & .7453 \\
Durante el último mes le costó más de lo habitual hacer sus quehaceres diarios a causa de & & \\
su salud física & -.1625 & .7449 \\
Durante el último mes tuvo que disminuir el tiempo dedicado a sus quehaceres diariosa & & \\
$\quad$ causa de un problema emocional (como estar triste, deprimido o nervioso) & .3006 & .7102 \\
Durante el último mes hizo menos delo que hubiera querido hacer a causa de un & & \\
problema emocional (como estar triste, deprimido o nervioso) & -.0774 & .7343 \\
Durante el último mes hizo sus quehaceres diarios con menos cuidado que el de & & \\
$\quad$ costumbre a causa de un problema emocional (como estar triste, deprimido o nervioso) & .0995 & .7223 \\
Tuvo dolor en alguna parte del cuerpo durante el último mes? & -.1961 & .7400 \\
Durante el último mes icuánto tiempo se sintió lleno de vida? & .3294 & .7084 \\
Durante el último mes ¿cuánto tiempo estuvo muy nervioso? & .1066 & .7215 \\
Durante el último mes ¿cuánto tiempo se sintió tan bajoneado que nada le subía el ánimo? & .3955 & .7060 \\
Durante el último mes icuánto tiempo se sintió calmado y tranquilo? & .6294 & .7014 \\
Durante el último mes icuánto tiempo tuvo mucha energía? & .3990 & .7087 \\
Durante el último mes se sintió desanimado y triste? & .3992 & .7077 \\
Durante el último mes icuánto tiempo se sintió agotado? & .2807 & .7118 \\
Durante el último mes ¿cuánto tiempo se sintió feliz? & .4298 & .7020 \\
Durante el último mes ¿cuánto tiempo se sintió cansado? & .2670 & .7127 \\
Durante el último mes su salud física o sus problemas emocionales, le han dificultado & & \\
sus actividades sociales tales como visitar a los amigos, familiares o salir a comprar? & -.2014 & .7361 \\
Cree que se enferma más fácilmente que otras personas? & .2617 & .7127 \\
Está tan sano como cualquier otras persona & .3856 & .7058 \\
Cree que su salud va ha estar más mala que como está ahora & .3120 & .7095 \\
Su salud es excelente & .1362 & .7202 \\
\hline
\end{tabular}

Al realizar el análisis de cada subescala podemos observar que en la escala de Salud Física, compuesta por 10 ítemes, el que se re fiere a "Por su salud actual se le hace difícil agacharse 0 arrodillarse" es el quetiene la correlación más baja. Si este ítem es eliminado el Alpha de Cronbach subea 0.74 , sin embar- go ésteesel único ítem quemide esta función motora, pese a que ésta puede verse interferidapor el efecto de algunos neurolépticos usados en el tratamiento de la esquizofrenia y a la edad de las personas que constituían la muestra, creemos que es aconsejable mantenerlo. 
Tabla 3. Estadísticos total-elemento subescala Salud Física.

\begin{tabular}{|l|c|c|c|c|c|}
\hline & $\begin{array}{c}\text { M edia de la } \\
\text { escalasi } \\
\text { se elimina } \\
\text { el elemento }\end{array}$ & $\begin{array}{c}\text { Varianza de } \\
\text { la escalasi } \\
\text { se elimina } \\
\text { el elemento }\end{array}$ & $\begin{array}{c}\text { Correlación } \\
\text { elemento-total } \\
\text { corregida }\end{array}$ & $\begin{array}{c}\text { Correlación } \\
\text { múltipleal } \\
\text { cuadrado }\end{array}$ & $\begin{array}{c}\text { Alfa de } \\
\text { Cronbach } \\
\text { si se elimina } \\
\text { el elemento }\end{array}$ \\
\hline pf3 & 20,00 & 21,776 &, 199 &, 243 &, 659 \\
pf4 & 19,45 & 20,353 &, 410 &, 335 &, 627 \\
pf5 & 19,48 & 19,620 &, 460 &, 433 &, 615 \\
pf6 & 19,97 & 19,907 &, 495 &, 374 &, 614 \\
pf7 & 19,42 & 20,083 &, 396 &, 343 &, 627 \\
pf8 & 19,80 & 19,734 &, 423 &, 443 &, 621 \\
pf9 & 19,20 & 19,530 &, 502 &, 370 &, 610 \\
(pf10) & $\mathbf{1 9 , 1 4}$ & $\mathbf{1 6 , 2 0 4}$ &, $\mathbf{2 1 5}$ & $\mathbf{, 1 2 2}$ &, $\mathbf{7 4 0}$ \\
pf11 & 19,82 & 21,273 &, 227 &, 171 &, 655 \\
pf12 & 18,98 & 20,898 &, 428 &, 305 &, 630 \\
\hline
\end{tabular}

En el análisis de la subescala Rol Físico el ítemesqueserefierea“D uranteel último mes tuvo que reducir el tiempo dedicado al trabajo 0 a sus actividades diarias a causa de su salud física" es el que tiene la correlación más baja, lo que puede deberse en parte a que los usuario desarrollan rutinas bastante fijas en loslugares donde viven por lo que tienen po- cas opciones de modificar sus actividades lo que concuerda con lo encontrado por Leibey Kallert (2001). Al eliminar este ítem el coeficienteAlpha aumenta a 0.78, sin embargo debemos considerar su inclusión en poblaciones que no tengan estas restricciones en las actividades de la vida diaria.

Tabla 4. Estadísticos total-elemento subescala Rol Físico.

\begin{tabular}{|l|c|c|c|c|c|}
\hline & $\begin{array}{c}\text { Media dela } \\
\text { escala si } \\
\text { se elimina } \\
\text { el elemento }\end{array}$ & $\begin{array}{c}\text { Varianza de } \\
\text { la escala si } \\
\text { se elimina } \\
\text { el elemento }\end{array}$ & $\begin{array}{c}\text { Correlación } \\
\text { elemento-total } \\
\text { corregida }\end{array}$ & $\begin{array}{c}\text { Correlación } \\
\text { múltipleal } \\
\text { cuadrado }\end{array}$ & $\begin{array}{c}\text { Alfa de } \\
\text { Cronbach } \\
\text { si se elimina } \\
\text { el elemento }\end{array}$ \\
\hline rp13 & 4,41 & 1,592 &, 587 &, 357 &, 597 \\
rp14 & 4,55 & 1,516 &, 522 &, 470 &, 629 \\
(rp15) & $\mathbf{4 , 6 0}$ & $\mathbf{1 , 6 7 2}$ & $\mathbf{, 3 0 0}$ & $\mathbf{, 1 5 0}$ & $\mathbf{7 8 0}$ \\
rp16 & 4,57 & 1,514 &, 635 &, 533 &, 566 \\
\hline
\end{tabular}

El análisis de la subescala Vitalidad y la correlación de sus ítemes muestra que ninguno de ellos tiene un impacto significativo sobre el índice de consistencia interna. Esta situación puededeberse a que la vitalidad de- finida para este instrumento como "Sentimiento de energía y vitalidad, frente al sentimiento de cansancio y agotamiento", está en sí misma comprometida en un gran número de personas que presentan alogia, aplana- 
miento afectivo, anhedoniay abulia como síntomas negativos residuales en el curso de la esquizofrenia crónica (Ashok, M alla, Jatinder, Takhar, Ross, Norman, M anchanda, Cortese, Haricharan, Verdi yA hmed, 2002). Es por esto que nos parece que esta escala no es representativa para evaluar esta función en personas con cuadros sicóticos de larga duración.

Tabla 5. Estadísticos total-elemento subescala Vitalidad.

\begin{tabular}{|c|c|c|c|c|c|}
\hline & $\begin{array}{c}\text { Media dela } \\
\text { escala si } \\
\text { se elimina } \\
\text { el elemento }\end{array}$ & $\begin{array}{c}\text { Varianza de } \\
\text { la escala si } \\
\text { se elimina } \\
\text { el elemento }\end{array}$ & $\begin{array}{c}\text { Correlación } \\
\text { elemento-total } \\
\text { corregida }\end{array}$ & $\begin{array}{c}\text { Correlación } \\
\text { múltipleal } \\
\text { cuadrado }\end{array}$ & $\begin{array}{c}\text { Alfa de } \\
\text { cronbach } \\
\text { si se elimina } \\
\text { el elemento }\end{array}$ \\
\hline vt23 & 6,09 & 2,798 &, 185 &, 128 &, 293 \\
vt27 & 6,06 & 2,894 &, 111 &, 116 &, 382 \\
vt29 & 5,85 & 2,620 &, 251 &, 290 &, 214 \\
vt31 & 6,12 & 2,904 &, 212 &, 284 &, 266 \\
\hline
\end{tabular}

Una situación similar es la que ocurrecon el análisis de la sub escala Salud M ental, la correlación de sus ítemes y el impacto sobre el índice de consistencia interna. Para efectos de este instrumento la Salud M ental General incluye la depresión, la ansiedad, el control de la conducta, el control emocional y el efecto positivo en general. Todos estos aspectos pueden verse comprometidos en distintos grados según la complejidad del cuadro clínico en personas que presenten esquizofrenia crónica en distintas fases de su enferme- dad como lo demuestran Giner et al. (2001) y Karow, Moritz, Lambert y Schoder y Krausz (2005) queconstatan quelossíntomasafectivos menos sicóticos, como los depresivosy losansiosos influyen directamente sobre la calidad devida delosenfermos. Lo mismo ocurrecon la sintomatología residual presenteen pacientessicóticos que han recibido tratamiento con neurolépticos clásicos (D elamillieure et al., 2005). Es por esto que nos parece que esta escala no es representativa para evaluar esta función en este grupo particular de personas.

Tabla 6. Estadísticos total-elemento subescala Salud M ental General.

\begin{tabular}{|c|c|c|c|c|c|}
\hline & $\begin{array}{c}\text { Media dela } \\
\text { escala si } \\
\text { se elimina } \\
\text { el elemento }\end{array}$ & $\begin{array}{c}\text { Varianza de } \\
\text { la escala si } \\
\text { se elimina } \\
\text { el elemento }\end{array}$ & $\begin{array}{c}\text { Correlación } \\
\text { elemento-total } \\
\text { corregida }\end{array}$ & $\begin{array}{c}\text { Correlación } \\
\text { múltipleal } \\
\text { cuadrado }\end{array}$ & $\begin{array}{c}\text { Alfa de } \\
\text { Cronbach } \\
\text { si se elimina } \\
\text { el elemento }\end{array}$ \\
\hline mh24 & 9.2 & 3.693 &, 195 &, 052 &, 295 \\
mh25 & 9.12 & 3.291 &, 333 &, 257 &, 170 \\
mh26 & 9.22 & 4.011 &, 064 &, 157 &, 409 \\
mh28 & 9.36 & 3.907 &, 181 &, 307 &, 308 \\
Mh30 & 9.15 & 3.987 &, 135 &, 022 &, 343 \\
\hline
\end{tabular}


Si se re-evalúa la consistencia interna del Instrumento total eliminando las escalas de Salud M ental y Vitalidad el CoeficienteAlpha aumenta a 0.79 .

\section{CONCLUSIONES}

Según los resultados obtenidos del presente estudio, podemos concluir quelos instrumentos diseñados para población general requieren de su validación en poblaciones específicas y no necesariamente pueden ser utilizados si no se efectúan las modificaciones pertinentes. Este es el caso del SF-36, que si bien ha probado su utilidad en población general, no puede ser aplicado sin realizar modificaciones en poblaciones de personas con esquizofrenia, ya que los parámetros que mide no consideran los cambios producidos en las personas por la enfermedad misma como los secundarios al uso de fármacos neurolépticos.

Sin embargo, éstos se constituyen como elementos iniciales para la investigación y como punto de partida para la construcción de nuevos instrumentos más específicos y ajustados a las características de las poblaciones estudiadas.

La esquizofrenia es un cuadro crónico que puede ser invalidante para quienes lo padecen debido a las dificultades que presenta la reinserción social de los usuarios por la tradición intramural que tiene nuestro país en la atención de pacientes psiquiátricos.

En Chile, la investigación de este tema en poblaciones de enfermos mentales es escasa y Enfermería tiene una responsabilidad social frentea estetema, por esto contar con un instrumento validado para la realidad chilena, es relevante como herramienta de valoración y diagnóstico para diseñar estrategias de intervención y la posterior implementación de programas de cuidados de enfermería que se elaboren a partir de las necesidades de los usuarios, lo cual seenmarca en la línea de Promoción dela Salud y el Autocuidado y se ali- nea con los planteamientos del Plan Nacional de Salud M ental y Psiquiatría vigente.

Por otro lado, la Enfermería puede proyectarse liderando equipos de rehabilitación que contribuyan a mejorar la reinserción social, la calidad de vida, la autoestima y la autoeficacia de personas que históricamentehan sido discriminadas por los estigmas sociales que se asocian a la patología psiquiátrica crónica.

\section{REFERENCIAS}

Acuña, J. y Rojas, G ( 2000). Síntomas psiquiátricos y discapacidad en el Gran Santiago. Revista de la Sociedad Chilena de Salud M ental y Psiquiatría (4), 217-223

Alonso J.; Prieto L. y Antó, J.M . (1999). La versión española del SF-36 H ealth Survey (Cuestionario deSalud SF-36): Un instrumento para la medida de los resultados clínicos. M edicina Clínica Barcelona, 104, 771-776.

Ashok, K.; Jatinder, M.; Takhar, J.; Ross, M .; Norman, G.; M anchanda, R.; Cortese, L.; H aricharan, R.; Verdi, M. y Ahmed, R. (2002). Negativesymptoms in first episodenon-affective psychosis Acta Psychiatrica Scandinavica, 105 (6), 431-439.

Ayuso-M ateos, J.L.; Lasa, L.; Vázquez-Barquero, JL.; Oviedo, A. y Diez-M aneique, JF. (1999). Measuring health status in psychiatric community surveys: Internal and external validity of the Spanish version of the SF-36. Acta Psychiatrica Scandinavica, 99,26.

Brazier, J.E.; H arper, R.; Jones, N.M .; O'Cathain, A.; Thomas, K.J.; Usherwood, T. et al. (1992) Validating the SF-36 health survey questionnarie: New outcomes measure for primary care. British Medical Journal, 305, 160- 164

Björkman, T. y Hansson, L. (2002). Predictors of improvement in quality of life of long-term mentally ill individual sreceiving casemanagement. European Psychiatry, 17(1), 33-40.

Borthwick-Duffy, S.A. (1992). Q uality of life and quality of care in mental retardation . En L. Rowitz (Ed.), Mental Retardation in the year 2000, pp. 52-56. Berlin: Springer-Verlag.

Caron, J., Lecomte, Y., Stip, E. y Renaud, S. (2005). Predictors of quality of life in schizophrenia. 
Community Mental Health Journal, 41(4), 399-417.

Cavieres, A. y Valdevenito, M . (2005). Funcionamiento cognitivo y calidad de vida en la esquizofrenia. Revista ChilenadeN euro-psiquiatría, 43 (2), 97-108.

Cerda. E. (1984). Psicometría General (30 ed). Pag 52. Barcelona: Editorial Herber.

Delamillieure, P.; Ochoa-Torres, D.; Vasse, T.; Brazo, P.; Gourevitch, R.; Langlois, S. et al. (2005). The subjective quality of life in deficit and nondeficit schizophrenic patients European Psychiatry, 20(4), 346-348.

Dernovzek, M .Z.; Prevolnik Rupel, V.; Rebolj, M. y Tavcar, R. (2002). Calidad de vida y costes de tratamientosen pacientes ambulatorios esquizofrénicos tratados con neurolépticos depot. European Psychiatry Ed Esp, 9, 226-235.

Felce, D. y Perry, J. (1995). Q uality of life: It's D efinition and Measurement. Research in Developmental Disabilities,16, ( 1), 51 -74.

Giner, J.; Ibáñez, E; Cervera, S.; San Martín, A. y Caballero, R. (2001). Subjectiveexperienceand quality of lifein schizophreniaActasEspañolas de Psiquiatría. 29(4), 11-23.

Hernández, R.; Fernández, C. y Baptista, P. (1998). Metodología dela investigación ( $2^{2}$ ed) M éxico: Mc Graw-Hill.

Jenkinson, C.; Wright, L y Coulter, A. (1994) . Criterion validity and reliability of the SF-36in a population sample. Quality of Life Research, 3, 7-12.

Karow, A.; M oritz, S.; Lambert, M.; Schoder, S. y Krausz, M . (2005). PANSS syndromes and quality of lifein schizophrenia. Psychopathology, 38(6), 320-326.

Katschnig, H. (2000) Schizophrenia and quality of life. Acta Psiquiátrica Scandinava [Suppl], (407), 33-37.

Kebede, D.; Alem, A.; Shibre, T.; N egash, A.; Deyassa, N . y Beyero, T. (2004) Health related quality of life (SF-36) survey in Butajira, rural Ethiopia: Normative data and evaluation of reliability and validity. Ethiop Medical Journal, 42(4), 289-297.

Kerlinger, F. (1975). La confiabilidad. Cap. 26. En:
Kerlinger, F. (ed.) Investigación del comportamiento. M éxico: Nueva Editorial Interamericana S.A. de C.V.

Leibe, M . y Kallert, T.W. (2001). Integración social y calidad de vida de los pacientes esquizofrénicos en tipos de asistencia complementaria diferentes European Psychiatry Ed Esp, 8, 93-104.

M ajali Mahasned, S. (2001). Health perceptions and health behaviours of poor Jordanian women. Journal of American Nursing, 16 (1), 58-68.

M cH orney, C .A .y Ware, J.J. \& Raczek, A.E. (1993). TheM OS 36-Item Short-Form Health Survey (SF-36): II Psychometric and clinical tests of validity in measuring physical and mental health constructs. Medical Care, 31, 247-263.

M inisterio deSalud deChile(2000). Plan Nacional de Salud M ental, Santiago, Chile.

Organización Mundial de la Salud (1980). International classification of impairments, disabilities and handicaps: a manual of classification relating to theconsequences of disease. Génova, Italia: Wood J.

Padierna, A.; Q uintana, J.M .; Arostegui, I.; González, N .; H orcajo, M .J. y Etxebarría, Y. (2000). Evolución clínica y percepción de bienestar en lostrastornos alimentarios. Anales de Psiquiatría, 16 (10), 407-414.

Reine, G.; Simeoni, MC.; Auquier, P.; Loundou, A.; A ghababian, V. y Lancon, C. (2005). Assessing health-related quality of life in patients suffering from schizophrenia: acomparison of instruments. European Psychiatry, 20 (7), 510519.

Tunis, SL.; Croghan, TW.; H eilman, DK.; Johnstone, BM . y Obenchain, RL. (1999). Reliability, validity, and application of themedical outcomes study 36-item short-form health survey (SF36) in schizophrenic patients treated with olanzapine versus haloperidol. M edical Care, 37(7), 678-691.

Ware, J.E. y Sherbourne C.D. (1992). The MOS 36-item form health survey (SF-36): conceptual framework and item selection. Medical Care, 30, 473-483. 